\title{
Variation of algae scum in electro-coagulation-flotation (ECF) process
}

\author{
Liang Tian ${ }^{\mathrm{a}^{*}}, \operatorname{Tian}_{\mathrm{Xu}}{ }^{\mathrm{b}}$, Xiaoxiao $\mathrm{Lu}^{\mathrm{c}}$, Jinrui $\mathrm{Xu}^{\mathrm{d}}$ \\ Key Laboratory of Environmental Medicine and Engineering, Ministry of Education, \\ School of Public Health, Southeast University, Nanjing, 210009, China

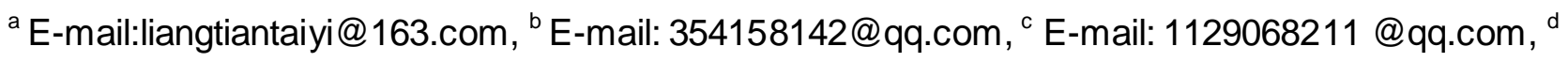 \\ E-mail: 1039995689@qq.com
}

Key words: algae scum; electro-coagulation-flotation; operating parameters; moisture content; slag water rate

Abstract. Algae scum, which is inevitable in the algae water separation process, should be harvested and forbidden to discharge into the water. This paper studied the variation of algae scum in electro-coagulation-flotation (ECF) process. All experiments were conducted in the homemade electro-coagulation-flotation device, and the operating parameters included electric current density and operating time. The results showed that the variation of algae scum is affected by the operating parameters. With increasing of the electric current density and prolongation of the operating time, the moisture content of algae scum decreases gradually. On the contrary, with increasing of the electric current density and prolongation of the operating time, the slag water rate increases gradually. And the values of the moisture content and the slag water rate are relatively lower than other technologies. Overall, the results showed that the electro-coagulation-flotation (ECF) is a well suitable technology for algae water separation.

\section{Introduction}

The occurrence of algae in reservoirs, lakes and rivers used as drinking water sources is a worldwide environmental health issue, due to the ability of some algal streamsto produce toxins, as well as taste and odour compounds, as secondary metabolites under particular conditions of growth [1]. Furthermore, the algae would also adversely affect the drinking water treatment process because of causing filter clogging and penetrating the filter $[2,3]$.

One possible solution is to separate algae from the medium (lakes, reservoir, estuaries, coasts, etc.) and there are several methods of separating algae such as salvage, sedimentation, centrifugation, coagulation, flocculation [4,5]. Although conventional methods are still the main treatment process for algae removal, due to the small size and low specific gravity, it is difficult to remove algae effectively [3]. What's more, the traditional methods are not safe barriers against algae and associated toxins [1].

Electro-coagulation-flotation (ECF), which seems to have more attractive than the conventional techniques for the treatment of algae-laden waters, runs greatly low energy consumption and safely $[4,6]$. Poelman [7] used ECF for microalgae recovery in drinking water treatment indicated removal efficiencies of $95 \%$ or more were easily obtained with different microalgae strains, and most important of all, energy consumption was as low as approximately $0.3 \mathrm{~kW}{ }^{-} \mathrm{h}^{\cdot} \mathrm{m}^{-3}$. Enrique [4] summarized the electro-flocculation, which does not damage the environment, is a suitable method for the eutrophication and the blooms of algae and cyanobacteria taking place in fluvial ecosystems. 
Algae scum, which is inevitable in the algae water separation process, should be harvested and forbidden to discharge into the water [8]. The fresh algae scum brings a series of problems, due to the higher moisture content [5]. So the researches on the algae scum are required in the EFC process. Consequently, the objectives of current study are to research the variation of algae scum.

\section{Materials and methods}

Preparation of the algal suspension. Microcystis aeruginosa (FACHB-905), one of the predominant algae species found in most eutrophic water bodies in China [6], provided from the Fresh Algae Culture Collection of the Institute of Hydrobiology, Chinese Academy of Sciences was used in this study. Biomass was cultivated on BG11 medium under general electric cool white fluorescent lamps at an average intensity of $150 \mathrm{umol}$ photons $/ \mathrm{m} 2 / \mathrm{s}$ with a 12 -h photoperiod, manually agitated three to four times everday [9]. The culture solution of Microcystis aeruginosa in the study was diluted by deionized water.

The ECF experimental device. The experiments were carried out in a glass flume with configuration of $25.5 \mathrm{~cm}$ (length) $\times 10 \mathrm{~cm}$ (width) $\times 30 \mathrm{~cm}$ (height) is schematically shown in Fig.1. Two aluminum plates with configuration of $25 \mathrm{~cm}$ (length) $\times 0.3 \mathrm{~cm}$ (width) $\times 25 \mathrm{~cm}$ (height) were kept $3 \mathrm{~cm}$ apart used as the electrode material with the effective area of $625 \mathrm{~cm}^{2}$. The electric current, obtained from the ammeter (VC97, Victor), was provided with a stabilized current supply (WYJ-0-60V/3A, China). Each experimental run was performed with 6L testing water with the initial $\mathrm{pH}$ of 7.0.

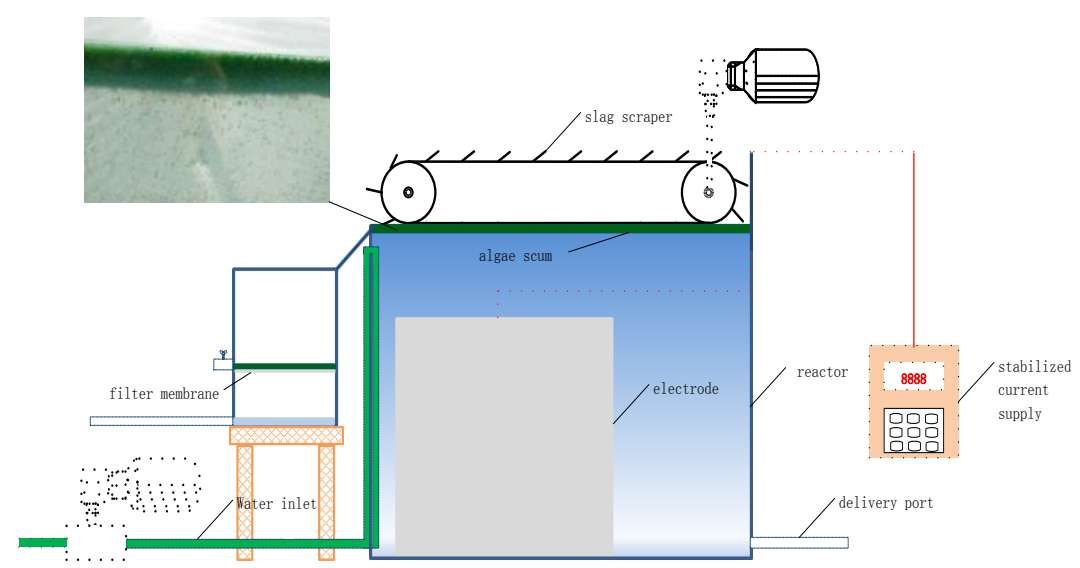

Fig.1 Sche matic diagram of the experimental device

Experimental procedure. All experiments were conducted in the homemade electro-coagulation-flotation device. And the initial algal concentrations ranged from $1.5 \times 10^{6} / \mathrm{L}$ to $1.5 \times 10^{9} / \mathrm{L}$. Each initial algal concentration was treated by four different electric current densities, respectively were $1 \mathrm{~mA} / \mathrm{cm}^{2}, 2 \mathrm{~mA} / \mathrm{cm}^{2}, 3 \mathrm{~mA} / \mathrm{cm}^{2}, 4 \mathrm{~mA} / \mathrm{cm}^{2}$. Operating times of the electric current density were determined at 5, 10, 15, $20 \mathrm{~min}$. Samples were taken at the water surface by slag scraper which was allowed to settle for $20 \mathrm{~min}$.

Measurements of algae scum. The analysis of moisture content of algae scum was carried out using the $103-105^{\circ} \mathrm{C}$ drying method by a box type resistance furnace control box (SX-12-10, China) [10]. We used the vernier caliper (PD-151, Pro skit) to measure the height of the algae scum and calculate the slag water rate.

Statistics. All curves were fitted through the program Origin 8.0. All of the data were obtained by repeating the experiments three times. 


\section{Results and Discussion}

Moisture content of algae scum. It has been established that the electric current density exerts a significant influence on algae removal in EFC process [6]. So the factor will also affect the moisture content of algae scum. In this investigation, the experiments for moisture content of algae scum were carried out with a wide range of electric current densities $\left(1-4 \mathrm{~mA} / \mathrm{cm}^{2}\right)$ at initial $\mathrm{pH}$ of 7 . As shown in Fig.2, the moisture content of algae scum decreased gradually with the operating time. Under the same conditions, the values of the electric current densities were greater, the moisture content of algae scum was lower. More interesting is that when the initial algal concentration is from $1.5 \times 10^{6} / \mathrm{L}$ to $1.5 \times 10^{9} / \mathrm{L}$, the moisture content of algae scum also decreased. It has been found that the moisture content of the algae scum in the floatation tank is higher than $99.5 \%$ [11]. But when the electric current density is higher than $2 \mathrm{~mA} / \mathrm{cm}^{2}$ and the operating time is longer than 10min, the moisture content of the algae scum in EFC process is lower than $99.5 \%$. Therefore, the significant advantages of ECF over air flotation are that the algae scum has lower moisture content and the algae removal is efficient.

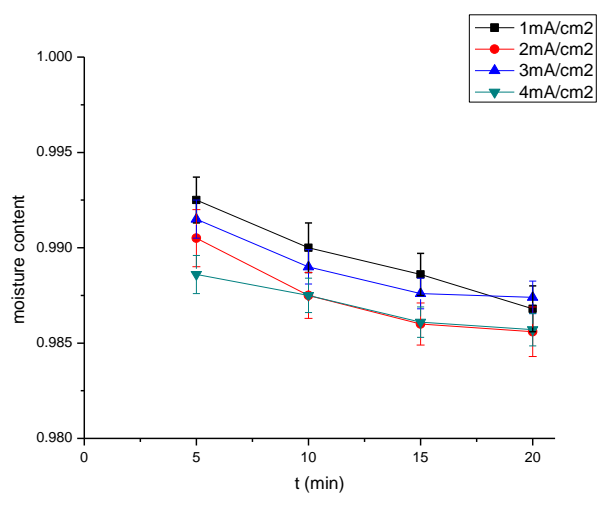

$1.5 \times 10^{9} / \mathrm{L}$

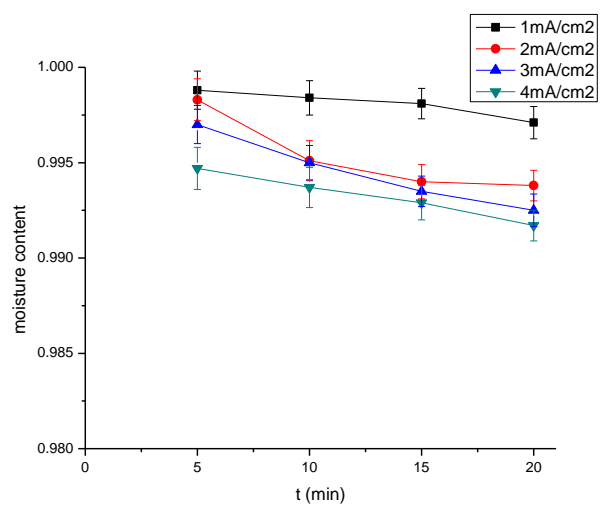

$1.5 \times 10^{7} / \mathrm{L}$

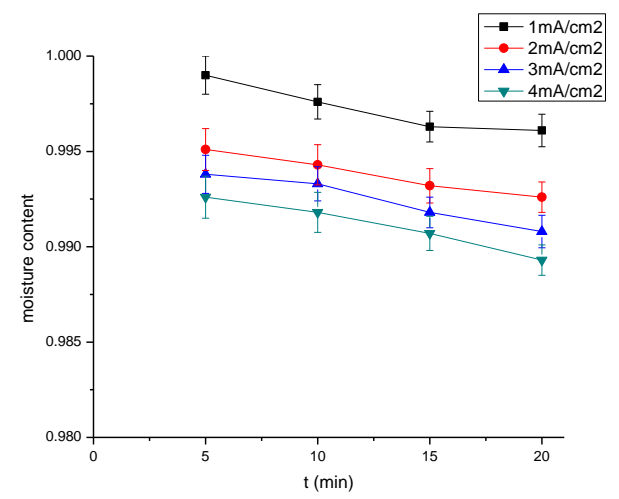

$1.5 \times 10^{8} / \mathrm{L}$

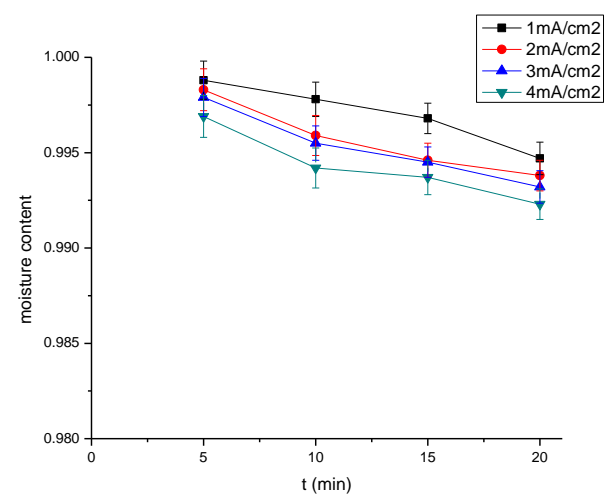

$1.5 \times 10^{6} / \mathrm{L}$

Fig.2 Variation of moisture content of algae scum with time at different electric current densities

Slag water rate. Fig.3 depicts the variation of slag water rate with time at different electric current densities. As can be seen from Fig.3, within the $20 \mathrm{~min}$, the slag water rate reached about $6 \%$ when the electric current density is $4 \mathrm{~mA} / \mathrm{cm}^{2}$. But when the electric current density turned to $1 \mathrm{~mA} / \mathrm{cm}^{2}$, 
the slag water rate reached about $1 \%$ after $20 \mathrm{~min}$. We can also learn from Fig.3 that, when the processing time extended to $15 \mathrm{~min}$, the curves of slag water rate tend to be sudden rise when the electric current density is $4 \mathrm{~mA} / \mathrm{cm}^{2}$. On the contrary, when the initial algal concentration is from 1.5 $\times 10^{6} / \mathrm{L}$ to $1.5 \times 10^{9} / \mathrm{L}$, the slag water rate increased. The mutative phenomenon of the slag water rate may be due to the changes of fractal dimension of the flocculation particles.

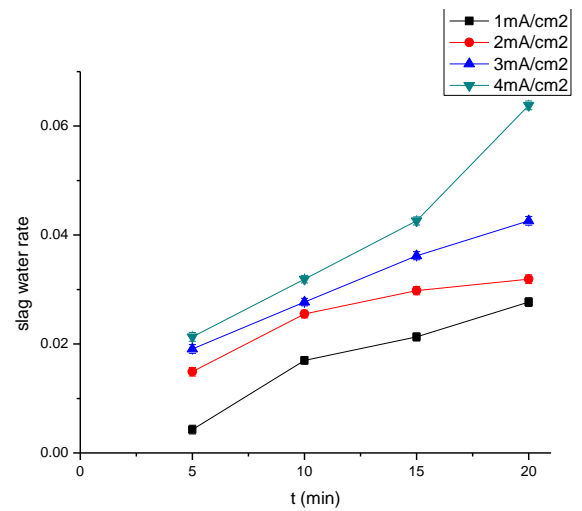

$1.5 \times 10^{9} / \mathrm{L}$

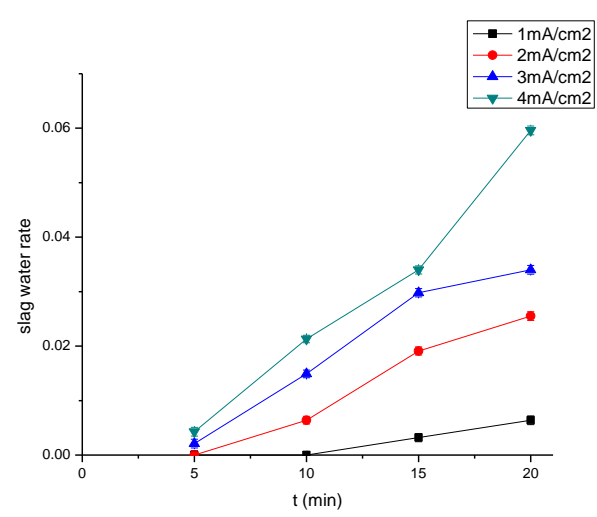

$1.5 \times 10^{7} / \mathrm{L}$

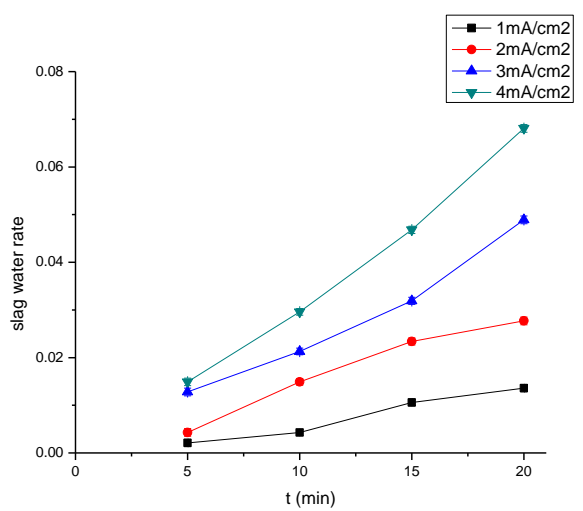

$1.5 \times 10^{8} / \mathrm{L}$

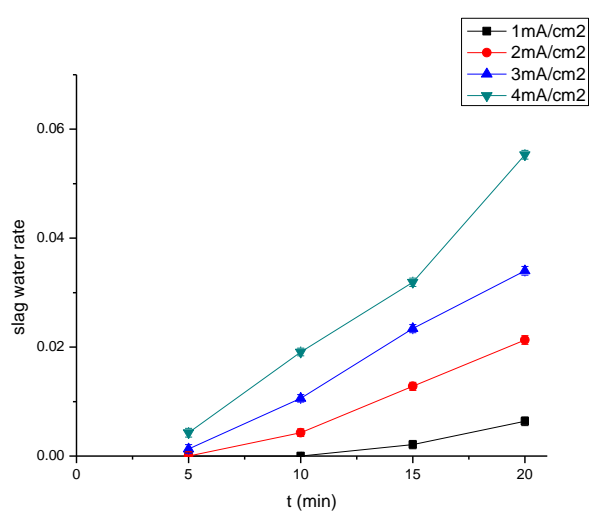

$1.5 \times 10^{6} / \mathrm{L}$

Fig.3 Variation of slag water rate with time at different electric current densities

\section{Conclusion}

The variation of algae scum in ECF process is affected by the operating parameters, such as electric current density and operating time. With increasing of the electric current density and prolongation of the operating time, the moisture content of algae scum decreases gradually. On the contrary, with increasing of the electric current density and prolongation of the operating time, the slag water rate increases gradually. And the values of the moisture content and the slag water rate are relatively lower than other technologies. Overall, the results showed that the EFC technology is well suitable process for algae water separation.

\section{Reference}

[1] M.R. Teixeira, V. Sousa, M.J. Rosa, Water Res.44(2010)3337-3344.

[2] J. Ma, W. Liu, Water Res.36(2002)871-8. 
[3]S. Gao, M. Du, J. Tian, J. Yang, J. Yang, F. Ma, J. Nan, Journal of Hazardous Materials.182(2010)827-834.

[4] E. Valero, X. Álvarez, Á. Cancela, Á. Sánchez, Bioresource Technology.187(2015)255-262.

[5]H.B. Xiong, Y.Y. Li, Q. Zhang, Chinese Journal of Environmental Engineering.02(2014) 599-604. (In Chinese)

[6]S. Gao, J. Yang, J. Tian, F. Ma, G. Tu, M. Du, Journal of Hazardous Materials.177(2010)336-343.

[7]E. Poelman, N. DePauw, B. Jeurissen, RESOURCES CONSERVATION AND REC YCLING.19(1997)1-10.

[8]Ministry of Housing and Urban-Rural Development. S .CJJ32(2011). (In Chinese)

[9] Y.J. Qiu, F. Rong, F. Yang, J.P. Li, H. Long, W. Wu, Fan, W,383-390(2012)3758-3762

[10] T.J. Geng, Nankai University.D.(2007) (In Chinese)

[11] S.P. Liu, Nankai University.D.(2007) (In Chinese) 